\title{
The Complex Structure of the Anterior White Commissure of the Human Brain: Fiber Dissection and Tractography Study
}

\author{
Safiye Çavdar ${ }^{1}$, Ayşegül Esen Aydın², Oktay Algin ${ }^{3,4}$, Seçkin Aydın ${ }^{5}$ \\ ${ }^{1}$ Koç University, School of Medicine, Department of Anatomy, Istanbul, Turkey. \\ ${ }^{2}$ Bakırköy Ruh ve Sinir Hospital, Department of Neurosurgery, Istanbul, Turkey. \\ ${ }^{3}$ Yıldırım Beyazıt University, Radiology Department, City Hospital, Ankara, Turkey and \\ ${ }^{4}$ National MR Research Center (UMRAM), Bilkent University, Ankara, Turkey \\ ${ }^{5}$ Okmeydani Training and Research Hospital, University of Health Sciences, Department of \\ Neurosurgery, Istanbul, Turkey.
}

Address for correspondence

Prof. Safiye Çavdar

Koç University,

School of Medicine,

Department of Anatomy,

34450 Sariyer

Istanbul Turkey

Fax No: 902163381073

E-mail: scavdar@ku.edu.tr

Funding: No funding was received for this study.

Conflict of interest: The authors declare that the research was conducted in the absence of any commercial or financial relationships that could be construed as a potential conflict of interest.

Ethical approval: All procedures performed in the studies involving human participants were approved by the ethical standards of the institutional Committee of Koç University.

Informed consent: Informed consent was obtained from all individual participants for the DTI part of the study. The brains used for the fiber dissection was donated to the Anatomy department for education and research purposes.

Key Words: Anterior commissure, commissural fibers, interconnection, fiber dissection, diffusion tensor tractography, human 


\section{Abstract}

OBJECTIVES: Commissural fibers are necessary for bilateral integration, body coordination, and complex cognitive information flow between the hemispheres. The anterior commissure (AC) has a complex architecture interconnecting areas of the frontal, temporal and occipital lobes. The present study aims to demonstrate the connections and the course of the anterior (ACa) and posterior (ACp) limb of the $\mathrm{AC}$ using fiber dissection and DTI technique in the human brain.

METHODS: Fiber dissection was performed in a stepwise manner from lateral to medial on 6 left hemispheres. The gray matter was decorticated and the ACa-ACp was exposed. The ACa and ACp tracts were demonstrated using a high-spatial-resolution DTI with a 3-tesla MR unit in 13 cases.

RESULTS: Using both techniques showed that, the AC has complex interconnections with large areas of the frontal (olfactory tubercles, anterior olfactory nucleus, olfactory bulb, and the orbital gyri), temporal (amygdaloidal nuclei, temporal and perirhinal cortex), and occipital (visual cortex) lobes. The ACp make up the major component of the AC and is composed of temporal and occipital fibers. We observed that these fibers do not make a distinct bundle; the temporal fibers joined the uncinate fasciculus and the occipital fibers joined the sagittal striatum to reach their targets.

CONCLUSION: Being aware of the course of the AC is important during transcallosal and interforniceal approaches to the $3^{\text {rd }}$ ventricle tumors and temporal lobe epilepsy surgery. The intermingling fibers of the AC can provide a better understanding of the unexplained deficit that may occur during regional surgery. 


\section{INTRODUCTION}

The various part of the brain and the spinal cord cross talks via; projection, association, and commissural fibers. Projection fibers connect the cerebral cortex to the brainstem and spinal cord. Association fibers connect brain regions within the same hemisphere and the commissural fibers connect corresponding regions of the left and right hemispheres of the brain. $^{1,2}$

Coordinated transfer of information between the two brain hemispheres is essential for bilateral integration and for better body coordination. ${ }^{3}$ The main commissural fibers are the corpus callosum (CC), anterior commissure (AC), hippocampal commissure (HiC), habenular commissure (HaC), supraoptic commissure (SoC), and posterior commissure (PC). Commissural fibers are categorized according to the brain structures they connect. The CC, $\mathrm{AC}$, and $\mathrm{HiC}$ are the telencephalic commissures, the $\mathrm{HaC}$, SoC, and $\mathrm{PC}$ are diencephalic commissures. $^{2,4}$

The AC embryologically develops in the lamina terminalis located at the anterior wall of the $3^{\text {rd }}$ ventricle at 7-8 weeks of gestation. ${ }^{5}$ Phylogenetically, the AC is the oldest of the commissures and contains approximately 3.5 million fibers in humans. ${ }^{2,6}$ Some mammals rely exclusively on the $\mathrm{AC}$ for interhemispheric transfer and others use a combination of both the $\mathrm{AC}$ and $\mathrm{CC} .{ }^{7}$ Due to the functional and anatomical difference of the $\mathrm{AC}$ in various species there is a great deal of controversy related to its course and connections. ${ }^{6,8,9}$

In humans, the AC lies rostral to the putamen and globus pallidus and the columns of the fornix and superior-anterior to the temporal horn of the lateral ventricle. ${ }^{10}$ The $\mathrm{AC}$ of humans consists of two limbs: a small anterior limbs (ACa) and a larger posterior (ACp) limb. The association of the ACa with olfaction is well-known. However, the connections and the course of the ACp is less relevant. The literature related to the connectivity and the course of the ACp is sparse and the findings are rather inconsistent. 
Experimental studies on animals and radiological studies in humans reported various neurophysiological disorders (schizophrenia and bipolar and other psychiatric disorders) related to the AC. ${ }^{11,12}$ Further, AC is an important interhemispheric structure in neurooncology and epilepsy surgery. Although different research techniques (imaging, dissection, and tract-tracing) have been used to understand the detailed architecture of the AC, however its exact course, function, and clinical relevance are not well understood in humans. In the present study, we aimed to demonstrate the connections and the course of the ACa and ACp in detail using fiber dissection and DTI technique in the human brain.

\section{METHODS}

\section{Fiber dissection technique}

In the present study, 6 left formalin-fixed brain hemispheres were used. The age of the cadavers ranged between 66-78 years-old (mean, 67 years) and was donated to the Anatomy department for education and research purposes. The brains were fixed with $10 \%$ formalin for at least 1 month. None had a grossly visible deformity of the central nervous system. After fixation, all specimens were frozen in water for at least 1 week at $-18^{0} \mathrm{C}$ to facilitate dissection, in accordance with the Klingler method. ${ }^{13}$ The dura mater, arachnoid membrane, and surface vessels were removed carefully under the microscope (Carl Zeiss AG) with X6X40 magnification using Zeiss Surgical Microscope (Carl Zeiss AG, Oberkochen, Germany) after at least 1 hour of thawing under tap water. The dissections were performed using both Rhoton dissectors and handmade thin, wooden spatulas. Between dissections, the specimens were kept in a $70 \%$ alcohol solution at room temperature. The dissections were performed in a stepwise manner from lateral to medial to reveal the 3D anatomy of the AC. Each stage of the dissections was recorded with digital photography.

\section{Diffusion tensor imaging (DTI) of the anterior commissure (AC)}

DTI data (9 males and 4 females, age range 18-50) were obtained from 13 healthy volunteers. 
The MRI exams were obtained using a 3T unit (Trio with Tim; Siemens Healthcare AG, Erlangen, Germany) with a 32-channel birdcage head coil. During MRI data acquisition, subjects were positioned with the head in the supine position in the MRI scanner. Fiber tracking was performed by deterministic tractography using seed points placed along the expected course of the white matter tracts of interest. For anatomical comparisons, we also included high-resolution anatomical imaging using a 5-minute T1-weighted axial magnetization-prepared rapid-acquisition gradient-echo sequence (repetition-time/echo-time $=2130 / 3.45$ milliseconds, flip angle $=8$, number of slices $=240$, field of view $=230 \times 230$ $\mathrm{mm}^{2}$, voxel size $=0.8 \times 0.8 \times 0.8 \mathrm{~mm}^{3}$ ). DTI and tractography data was obtained as mentioned below; DWI: TR $7750 \mathrm{~ms}$, TE: $89 \mathrm{~ms}$, FoV: $237 \mathrm{~mm}$, matrix: $128 \times 128$, slice thickness: $1.84 \mathrm{~mm}$, distance factor: $0 \%, 60$ slices, PAT: 2 with GRAPPA, voxel size: $1.8 \times 1.8 \times 1.8 \mathrm{~mm}^{3}$, diffusion encoding gradients: 60 for 12 cases and 256 for one male, b-values: $0-1000 \mathrm{~s} / \mathrm{mm}^{2}$, orientation: sagittal or transverse, phase encoding direction: anterior-posterior. For the demonstration of AC, diffusion seed points were placed at the interforniceal area for all cases on three planes anatomic images. Neuro 3D (Leonardo, Siemens Healthcare) and DSI Studio (www.labsolver.org) softwares were used for data analysis

\section{RESULTS}

\section{Fiber Dissection of the AC from lateral to medial}

The fiber dissection was initiated by removing the cortical gray matter from the lateral aspect of the left cerebral hemisphere. Then, the short association 'U' fibers that connect adjacent gyri were exposed (Fig.1a). After removing the 'U' fibers the long association fibers; the superior longitudinal fascicle 2 (SLF 2) and superior longitudinal fascicle 3 (SLF 3) were exposed in the perisylvian area (Fig. 1b). The SLF-2 extended from the angular gyrus to the middle part of the middle frontal gyrus and the SLF 3 extended between the supramarginal 
gyrus and the pars triangularis and pars opercularis of the inferior frontal gyrus (Fig. 1b). The arcuate fascicle was localized between the middle frontal and the inferior temporal gyri (Fig. 1c). The insula was exposed after removing SLF and the dorsal and ventral components of the arcuate fascicle. Furthermore, the temporal, frontal, and parietal operculum were removed for a better view of the insula.

The extreme capsule was revealed after removing the gray matter of the insula. After removing the extreme capsule, a thin layer of gray matter, claustrum, and external capsule fibers were exposed (Fig. 1c). The external capsule consisted of dorsal and ventral components. The fan-shaped dorsal component of the external capsule (claustrocortical fibers) extended from the claustrum to the corona radiata. The putamen and corona radiata were exposed by removing the dorsal component of the external capsule and the arcuate fascicle (Fig. 1d, e). The ventral component of the external capsule was associated with the inferior fronto-occipital fascicle (IFOF) and uncinate fascicle (Fig. e). These two fiber bundles were exposed after removing the ventral component of the external capsule. The IFOF extended to the pars opercularis and pars triangularis of the inferior frontal gyrus anteriorly, and crossed the arcuate fascicle, and joined the sagittal striatum posteriorly (Fig. 1e). The lenticular nucleus (putamen and globus pallidus) was exposed, and the putamen was localized at the most lateral part of the lentiform nucleus (Fig. e, f). After removing the putamen, the globus pallidus was exposed medially. The fiber bundle of the internal capsule were located medial to the lentiform nucleus. Superior to the lentiform nucleus the internal capsule fibers joined the claustrocortical fibers to form the corona radiata and formed the sagittal striatum at the posterior border of the lentiform nucleus (Fig. e, f).

The AC was localized at the anteroinferior aspect of the globus pallidus. The main bulk (body of the $\mathrm{AC}$ ) of the $\mathrm{AC}$ were twisted and intertwined like the strains of a rope and separated into anterior (ACa) and large posterior (ACp) limbs (Fig. 1f, g). The ACa left the 
main trunk at the level of the anterior perforated substance and olfactory tract and curved through the nucleus accumbens and extended into the orbital frontal gyrus (Fig. 1f, g). The substantia innominata was located between the anterior and posterior limbs of the AC (Fig. 1f, g). The ACp carried the major part of the AC and crossed the base of the lentiform nucleus from the anteromedial to the posterolateral aspect in the Gratiolet canal (Fig. 1e). The Gratiolet canal lied embedded in the gray matter of the putamen and the caudate nucleus, immediately anterior and below the anterior border of the globus pallidus. The fibers of the ACp fans out towards the anterior part of the temporal and occipital lobes (Fig. 1f, g). It courses along with the basal parts of the putamen, caudate nucleus, and below the anterior border of the globus pallidus (Fig. 1e). The number of fibers reaching the occipital lobe were more numerous than the fibers reaching the temporal lobe. The fibers extending into the temporal lobe were localized inferiorly and joined the fibers of the uncinate fascicle (Fig. $1 \mathrm{~g}$ ). The fibers coursing to the occipital lobe were located superiorly and were intermingled with IFOF and optic fibers and joined the sagittal striatum (Fig. 1g). These three fiber bundles were indistinctive in the sagittal striatum (Fig. 1g).

\section{DTI or tractography results}

We evaluated 13 cases using high-resolution 3D DTI data. The AC were demonstrated in different planes and bilaterally (Fig. 2a-d). The posterior fibers of the AC consisted of temporal and occipital fibers (Fig. 2 and 3). Similar to the fiber dissection observations the occipital fibers were more numerous compared to temporal fibers (Fig. 2e). The separation point of the occipital and temporal fibers from the ACp was rather constant, at the base of the globus pallidus (Fig. 2f). The body of the AC was closely related to the interforniceal area, thus discrimination and separation of these structures were difficult (Fig. 3). 


\section{DISCUSSION}

Although the exact function of the commissural fibers has long been debated, however, the common consensus is that these fibers are necessary for bilateral integration, better body coordination, and complex cognitive information flow between the brain hemispheres. The interconnection of the ACa with the olfactory system is well-known and is similar in many species. ${ }^{14}$ However, the course of the ACp is complex and interconnects larger territories than expected.

The commissural fibers are guided across the midline to reach their targets in the contralateral hemisphere via complex cellular and molecular mechanisms during embryological development. ${ }^{15}$ Thus, if the underlying mechanisms regulating the guidance of commissural fibers fail, pathological dysgenesis of one or more commissures occurs. ${ }^{16,17}$ Robichaux et al. ${ }^{18}$ observed partial and complete agenesis of the $\mathrm{CC}$, as well as $\mathrm{AC}$ misprojection phenotypes in truncated EphB1/2 receptors in mice that lack intracellular signaling domains. Due to the common embryological development and the molecular mechanism guiding the $\mathrm{CC}$ and the $\mathrm{AC}$, agenesis of the $\mathrm{CC}$ is often accompanied by the loss of the AC. ${ }^{2,19}$ However, there are also studies that show the compensation of the AC in the cases of agenesis of the $\mathrm{CC} .{ }^{20}$ In the study of Gerrish et al. ${ }^{21}$ the white matter volume of the $\mathrm{AC}$ and PC were examined in children with agenesis of the $\mathrm{CC}$ and were compared with control children, the results showed that the AC was significantly larger in volume in children with agenesis of the $\mathrm{CC}$. They concluded that in cases of $\mathrm{CC}$ agenesis, the AC may partially take over the interhemispheric connection function normally undertaken by the $\mathrm{CC}$, compensating for the lack of interhemispheric communication. Furthermore, studies have shown that individuals with congenital agenesis of the $\mathrm{CC}$ frequently exhibit less severe symptoms of disconnection syndrome compared with patients that underwent surgical transection of the CC during life. ${ }^{22-25}$ Thus, the congenital agenesis of the CC may permit 
developmental adaptive reorganization. However, studies have shown that CC cannot compensate for the interhemispheric communications of the $\mathrm{AC}$, thus the $\mathrm{AC}$ has both primary and vestigial projections to neocortical areas where the $\mathrm{CC}$ does not project. ${ }^{8,26}$

The course of the ACa fibers clearly shows its interconnections with the olfactory system (olfactory tubercles, anterior olfactory nucleus, and olfactory bulb) and the orbital gyri. Similar connections were demonstrated for cats, rats, and monkeys. ${ }^{8,26-28}$ However, the interconnection of the ACp is regarded to be more complex. The ACp does not reach the target structure in a unique bundle as the $\mathrm{ACa}$, instead, these fibers intermingle with other major white matter tracts to reach their target. The temporal fibers of the ACp joined the uncinated fasciculus to reach the temporal lobe (amygdaloidal nuclei, temporal and perirhinal cortex). The occipital fibers of the ACp joined the IFOF, optic tract, and the sagittal striatum. Early study using tract-tracing technique compared the connections of the AC of rat, cat, and monkey and reported that amygdaloid nuclei and the perirhinal and neocortices connections showed variations among species. ${ }^{28}$ The ACp interconnects regions related to higher-order brain functions via its amygdala and neocortical connections. Thus, phylogenetically, the ACa can be regarded as archi and the ACp is neo part of the AC.

Studies reported the relations of the AC pathologies with neurophysiological disorders. ${ }^{14,29}$ An experimental study using marmoset (South American) monkey compared the midsagittal sizes of the $\mathrm{AC}$ and $\mathrm{CC}$ exposed to valproic acid (display social recognition deficit in adulthood) with the control group using 7-Tesla MRI and found that the size of both $\mathrm{CC}$ and $\mathrm{AC}$ in the valproic acid-exposed group was significantly smaller. ${ }^{29}$ Furthermore, structural anomalies of the AC were reported in patients with bipolar and anxiety disorders, depression, and especially schizophrenia. ${ }^{14}$ Schizophrenia in particular is thought to result from a dysfunctional integration or disconnection within the brain. ${ }^{30}$ Oxidative stress and redox dysregulation have been proposed to play a role in the pathophysiology of psychiatric 
disorders. A study using a preclinical model of redox dysregulation showed reduced fractional anisotropy in the AC accompanied by reduced conduction velocity in fastconducting fibers of the ACp in a mouse model. ${ }^{12}$ In addition, behavioral studies using diffusion MRI showed that repeated exposure to an inescapable stressor in rats leads to microstructural white matter changes including the AC. ${ }^{31}$ The present study clearly shows the connections of the ACp with the amygdala and neocortex which may contribute to the modulation of emotional processing and complex cognitive functions.

The location, course, and function of the AC are surgically important and should be well known during the various approaches to the $3^{\text {rd }}$ ventricle. The anterior transcallosal and interforniceal approach are most frequently used to reach $3^{\text {rd }}$ ventricle tumors. Thus the close relations of the AC with the anterior horn of the $3^{\text {rd }}$ ventricle and fornix should be well known (see fig. 1g). However, the dislocation of the AC should be kept in mind, the dislocation of the AC was reported in more than a third of the cases $(38 \%){ }^{2}$ The anterior temporal lobectomy is one of the surgical approaches used in temporal lobe epilepsy. Temporal stem incision is required during this surgery. The temporal stem is a white matter bridge between temporal and frontal lobes and basal nuclei which includes ACp, uncinate fasciculus, IFOF, optic radiations, and inferior thalamic fibers. Studies have shown that visual defects and cognitive disorders observed in patients after temporal lobectomy. ${ }^{32,33}$ Therefore, the close relationship of the AC with the temporal stem should be kept in mind and special care should be taken to minimally damage these fibers during anterior temporal lobectomy surgery. ${ }^{34-37}$

\section{CONCLUSION}

The recent advances in modern imaging techniques, 3-tesla high-resolution MRI and DTI, have provided a noninvasive powerful tool in medical diagnosis and research related to the white matter connectivity of the brain. Thus, being aware of the normal anatomy of the AC 
can enable clinicians to improve the diagnostic accuracy and surgical options of many devastating neurological diseases.

\section{REFERENCE}

1. Rhoton Jr, AL. The cerebrum. Neurosurgery. 2007;61(1 Suppl):37-118 [discussion 118-9].

2. Raybaud C. The corpus callosum, the other great forebrain commissures, and the septum pellucidum: anatomy, development, and malformation. Neuroradiology. 2010;52(6):447-77.

3. Moldrich RX, Gobius I, Pollak T, Zhang J, Ren T, Brown L, Mori S, De Juan Romero C, Britanova O, Tarabykin V, Richards LJ. Molecular regulation of the developing commissural plate. Comp Neurol. 2010;518(18):3645-61.

4. Van der Knap LJ, Van der Ham IJ. How does the corpus callosum mediate interhemispheric transfer? A review. Behav Brain Res. 2011;223(1):211221.

5. Cho KH, Murakami G, Rodríguez-Vázquez JF. Early fetal development of the anterior commissure. Pediatr Neurol. 2013;8(1):56-8.

6. Guénot M. Transfert interhémisphérique et agénésie du corps calleux. Capacités et limites de la commissure blanche antérieure. Neurochirurgie (Paris). 1998;44(Suppl 1):113-115.

7. Ashwell KWS. Anterior commissure versus corpus callosum: A quantitative comparison across mammals. Zoology (Jena). 2016;119(2):126-136.

8. Di Virgilio G, Clarke S, Pizzolato G, Schaffner T. Cortical regions contributing to the anterior commissure in man. Exp Brain Res. 1999;124:1-7.

9. Lamantia AS, Rakic P. Cytological and quantitative characteristics of four cerebral commissures in the rhesus monkey. J Comp Neurol. 1990;291:520-537.

10. Pescatori L, Tropeano MP, Manfreda A, Delfini R, Santoro A. Three-dimensional anatomy of the white matter fibers of the temporal lobe: surgical implications. World Neurosurgery. 2017;100:144-158.

11. Wilde EA, Bigler ED, Haider JM, Chu Z, Levin HS, Li X, Hunter JV. The vulnerability of the anterior commissure in moderate to severe pediatric traumatic brain injury. $J$ Child Neurol. 2006;21(9):769-776. 
12. Corcoba A, Steullet P, Duarte JM, Van de Looij Y, Monin A, Cuenod M, Gruetter R, Do KQ. Glutathione deficit affects the integrity and function of the fimbria/fornix and anterior commissure in mice: relevance for schizophrenia. Int J Neuropsychopharmacology. 2015;19(3):1-11.

13. Ludwig E, Klingler J. Atlas Cerebri Humani. 1956 Basel, S. Karger.

14. Lavrador JP, Ferreira V, Lourenço M, Alexandre I, Rocha M, Oliveira E, Kailaya-Vasan A, Neto L. White-matter commissures: a clinically focused anatomical review. Surg Radiol Anat. 2019;41(6):613-624.

15. Egea J, Klein R. Bidirectional Eph-ephrin signaling during axon guidance. Trends Cell Biol. 2007;17:230-238.

16. Sisodiya SM, Free SL, Williamson KA, Mitchell TN, Willis C, Stevens JM, Kendall BE, Shorvon SD, Hanson IM, Moore AT, van Heyningen V. PAX6 haploinsufficiency causes cerebral malformation and olfactory dysfunction in humans. Nat Genet. $2001 ; 28(3): 214-6$.

17. Abouzeid H, Youssef MA, ElShakankiri N, Hauser P, Munier FL, Schorderet DF. PAX6 aniridia and interhemispheric brain anomalies. Mol Vis. 2009;15:2074-83.

18. Robichaux MA, Chenaux G, Ho HY, Soskis MJ, Greenberg ME, Henkemeyer M, Cowan CW. EphB1 and EphB2 intracellular domains regulate the formation of the corpus callosum and anterior commissure. Dev Neurobiol. 2016;76(4):405-20.

19. Raybaud C, Girard N. Malformations of the telencephalic commissures. Pediatric Neuroradiology. 2005;41-69.

20. Siffredi V, Wood AG, Leventer RJ, Vaessen M, Mcllroy A, Anderson V, Vuilleumier P, Spencer-Smith MM. Anterior and posterior commissures in agenesis of the corpus callosum: Alternative pathways for attention processes? Cortex. 2019;121:454-467.

21. Gerrish AC, Thomas AG, Dineen RA. Brain white matter tracts: functional anatomy and clinical relevance. Semin Ultrasound CT MRI. 2014;35(5):432-444.

22. Brown WS, Thrasher ED, Paul LK. Interhemispheric Stroop effects in partial and complete agenesis of the corpus callosum. J Int Neuropsychol Soc. 2001;7(3):302-11.

23. Chiarello C. A house divided? Cognitive functioning with callosal agenesis. Brain Lang. 1980;11(1):128-58. 
24. Paul LK, Brown WS, Adolphs R, Tyszka JM, Richards L, Mukherjee P, Sherr EH. Agenesis of the corpus callosum: genetic, developmental and functional aspects of connectivity. Nat Rev Neurosci. 2007;8(4):287-99.

25. Paul LK. Developmental malformation of the corpus callosum: a review of typical callosal development and examples of developmental disorders with callosal involvement. J Neurodev Disord. 2011;3(1):3-27.

26. Patel MD, Toussaint N, Charles-Edwards GD, Lin JP, Batchelor PG. Distribution and fibre field similarity mapping of the human anterior commissure fibres by diffusion tensor imaging. MAGMA. 2010;23(5-6):399-408.

27. Jouandet ML. Neocortical and basal telencephalic origins of the anterior commissure of the cat. Neuroscience. 1982;7:1731-1752.

28. Jouandet ML, Gazzaniga MS. Cortical field of origin of the anterior commissure of the rhesus monkey. Exp Neurol. 1979;66(2):381-97.

29. Mimura K, Oga T, Sasaki T, Nakagaki K, Sato C, Sumida K, Hoshino K, Saito K, Miyawaki I, Suhara T, Aoki I, Minamimoto T, Ichinohe N. Abnormal axon guidance signals and reduced interhemispheric connection via anterior commissure in neonates of marmoset ASD model. Neuroimage. 2019;15;195:243-251.

30. Friston KJ, Frith CD. Schizophrenia: a disconnection syndrome? Clin Neurosci. 1995;3:89-97.

31. Magalhães R, Bourgin J, Boumezbeur F, Marques P, Bottlaender M, Poupon C, Djemaï B, Duchesnay E, Mériaux S, Sousa N, Jay TM, Cachia A. White matter changes in microstructure associated with a maladaptive response to stress in rats. Trans/ Psych. 2017;24;7(1):e1009.

32. Uribe JS1, Vale FL. Limited access inferior temporal gyrus approach to mesial basal temporal lobe tumors. J Neurosurg. 2009;110(1):137-46.

33. Nascimento FA, Gatto LA, Silvado C, Mäder-Joaquim MJ, Moro MS, Araujo JC Anterior temporal lobectomy versus selective amygdalohippocampectomy in patients with mesial temporal lobe epilepsy. Arq Neuropsiquiatr. 2016;74(1):35-43.

34. Girvin JP. Temporal lobectomy. In: Apuzzo ML (ed). Neurosurgical Aspects of Epilepsy (Neurosurgical Topics). Park Ridge, IL: American Association of Neurological Surgeons. 1990;157-170. 
35. Olivier A. Surgery of epilepsy: overall procedure. In: Apuzzo MLJ (ed). Neurosurgical Aspects of Epilepsy (Neurosurgical Topics). Park Ridge, IL: American Association of Neurological Surgeons. 1990;117-148.

36. Spencer DD, Ojemann GA. Overview of therapeutic procedures. In: Engel J Jr (ed). Surgical Treatment of the Epilepsies, Philadelphia: Lippincott-Raven. 1996;455-471.

37. Choi CY, Han SR, Yee GT, Lee $\mathrm{CH}$. An understanding of the temporal stem. J Korean Neurosurg Soc. 2010;47(5):365-9.

\section{Legend to figures}

Figure 1: Fiber Dissection of the AC from lateral to medial. 1a. The cortical gray matter was removed and the short association ' $U$ ' fibers were exposed. 1b. The long association fibers superior longitudinal fascicle 2 (SLF 2) and superior longitudinal fascicle 3 (SLF 3) were exposed. 1c. The insular cortex was removed and the clastrum and internal capsule were exposed. 1d. The putamen and the corona radiate was exposed. 1e. The lenticular nucleus formed by putamen and globus pallidus was exposed and the close relation of the Gratiolet canal to the AC was exposed. 1f. The small anterior (ACa) and large posterior (ACp) limbs of the AC are exposed and its relations with nucleus accumbens, substantia innominate, globus pallidus, caudate nucleus were revealed. 1g. Fine fiber dissection of AC and its relations with nearby structures and the ACp showing its temporal and occipital fibers.

Figure 2. The DTI images obtained with Leonardo software showing the posterior limb of the AC. 2a-e. Shows the DTI of the posterior limb of the AC in different planes. The temporal fibers on the right and occipital fibers on the left are demonstrated. 2e. The DTI shows a unilateral posterior limb of the AC. Note that the occipital fibers are more numerous than temporal fibers. 2f. The DTI showing the separation of the temporal and occipital fibers of the posterior limb of the $\mathrm{AC}$ at the base of the globus pallidus. 
Figure 3. The AC tracts obtained with DSI studio software clearly show bilateral occipital and temporal branches of a 29-year old male (case no: 13 ). 
Figure(s)
Click here to download high resolution image

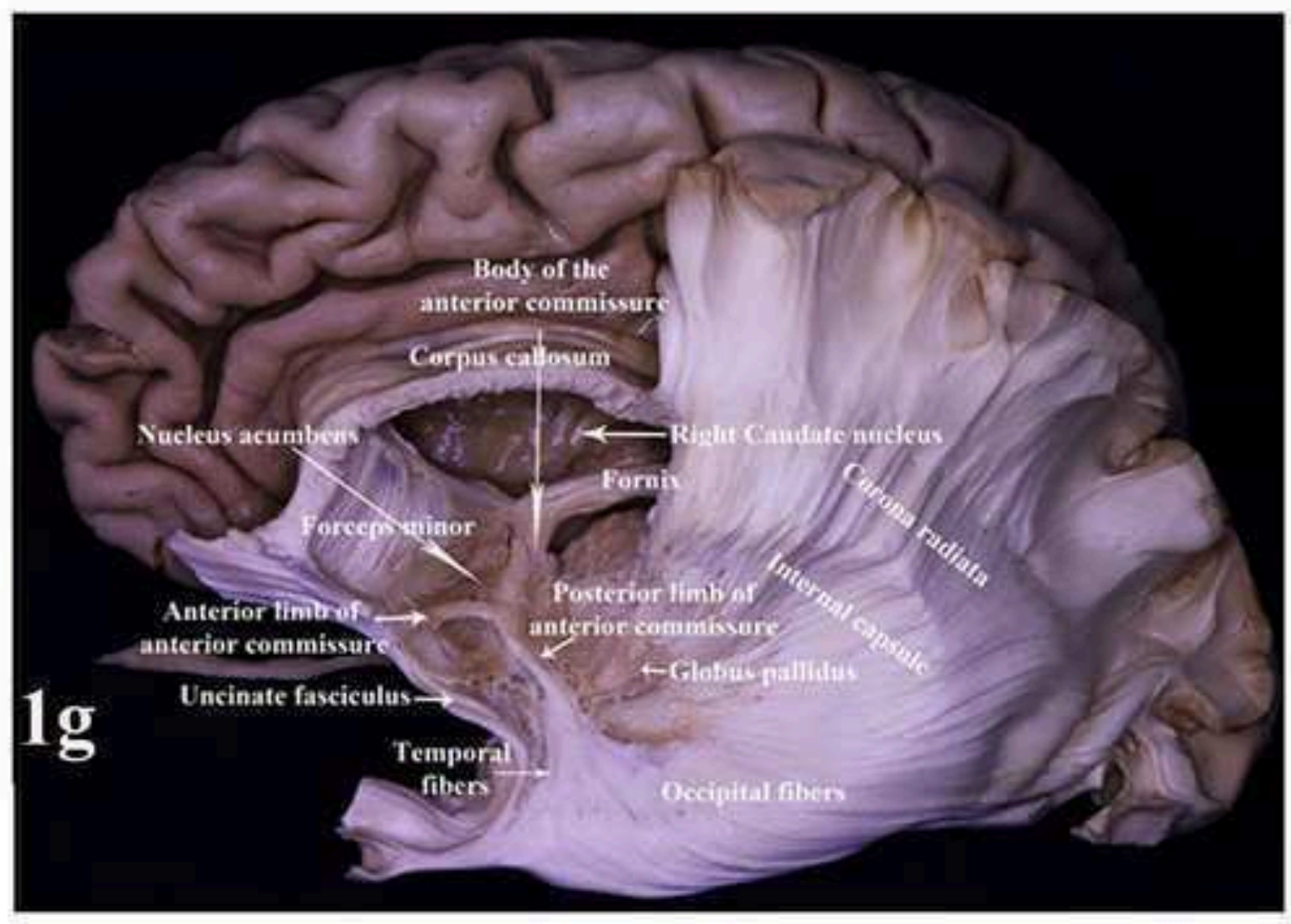



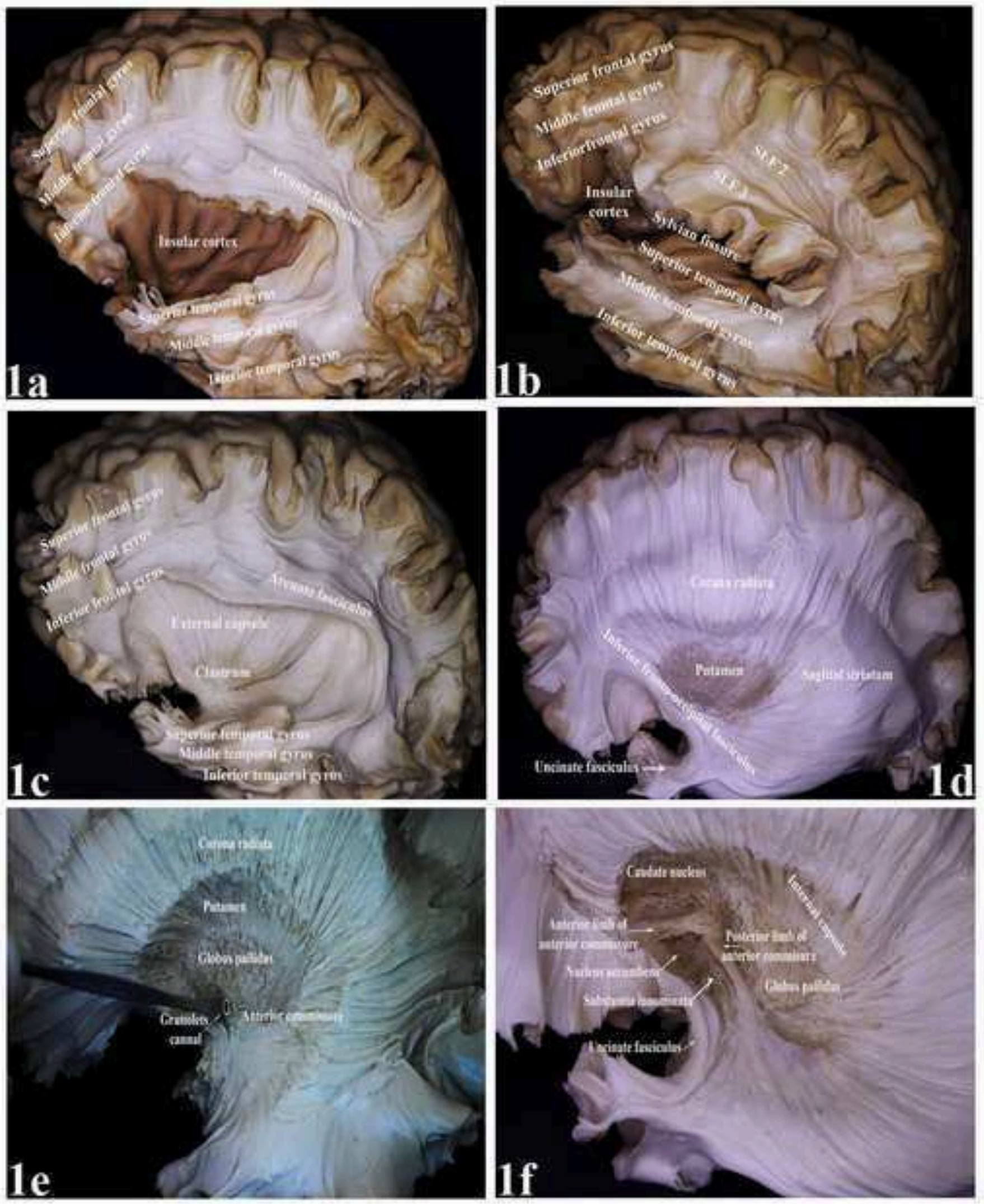


\section{Figure(s)}

Click here to download high resolution image
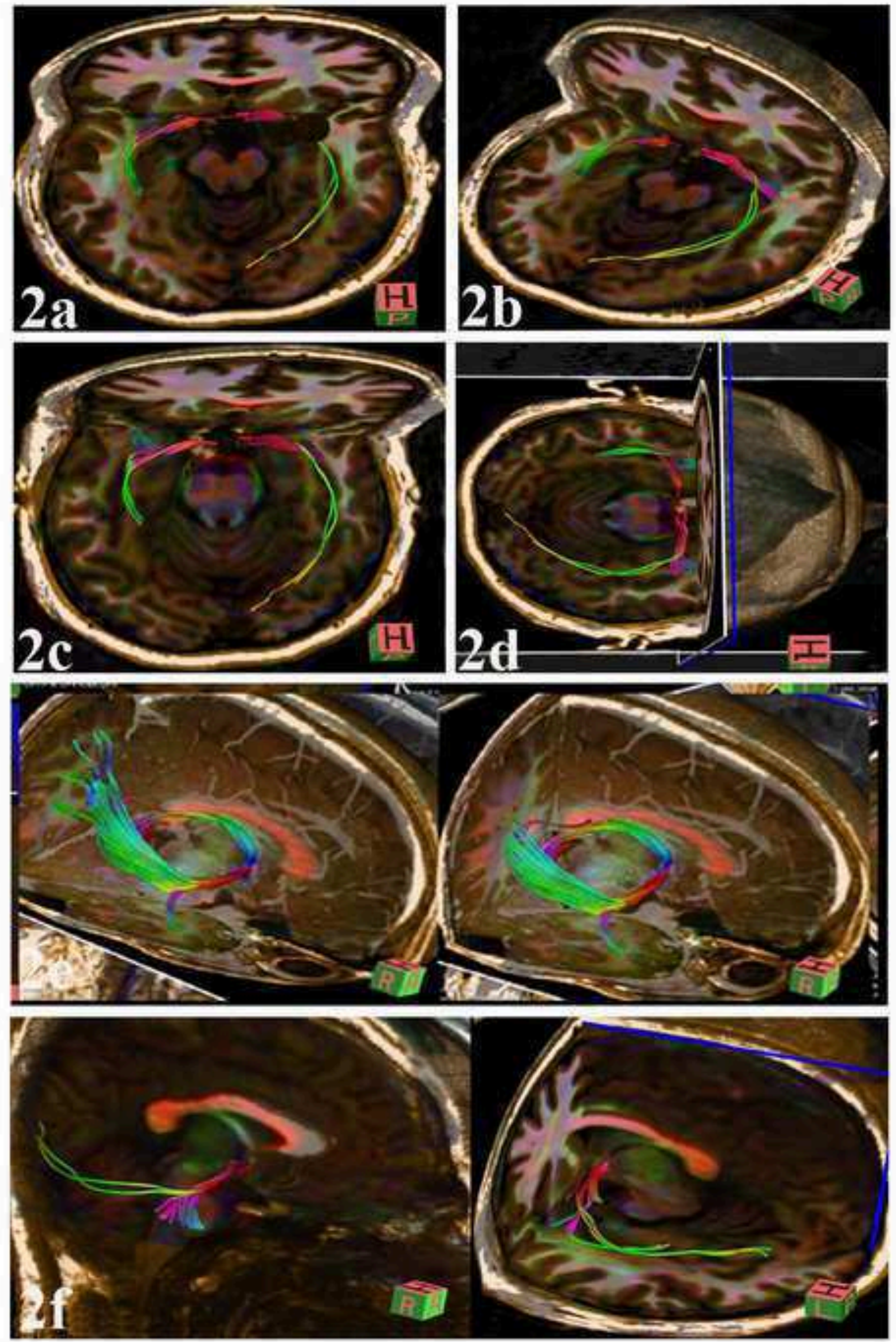


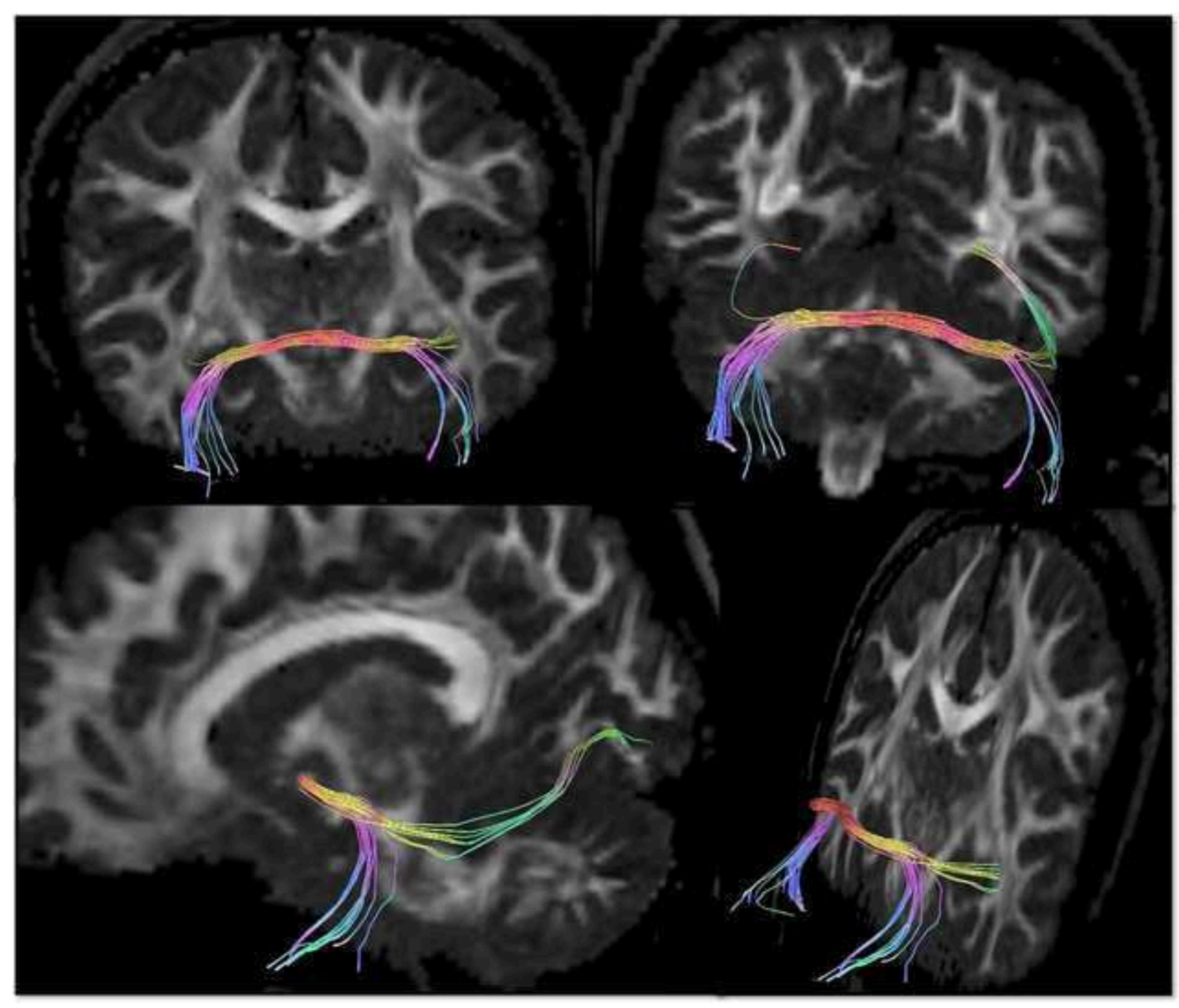

Fig. 3
Click here to download high resolution image
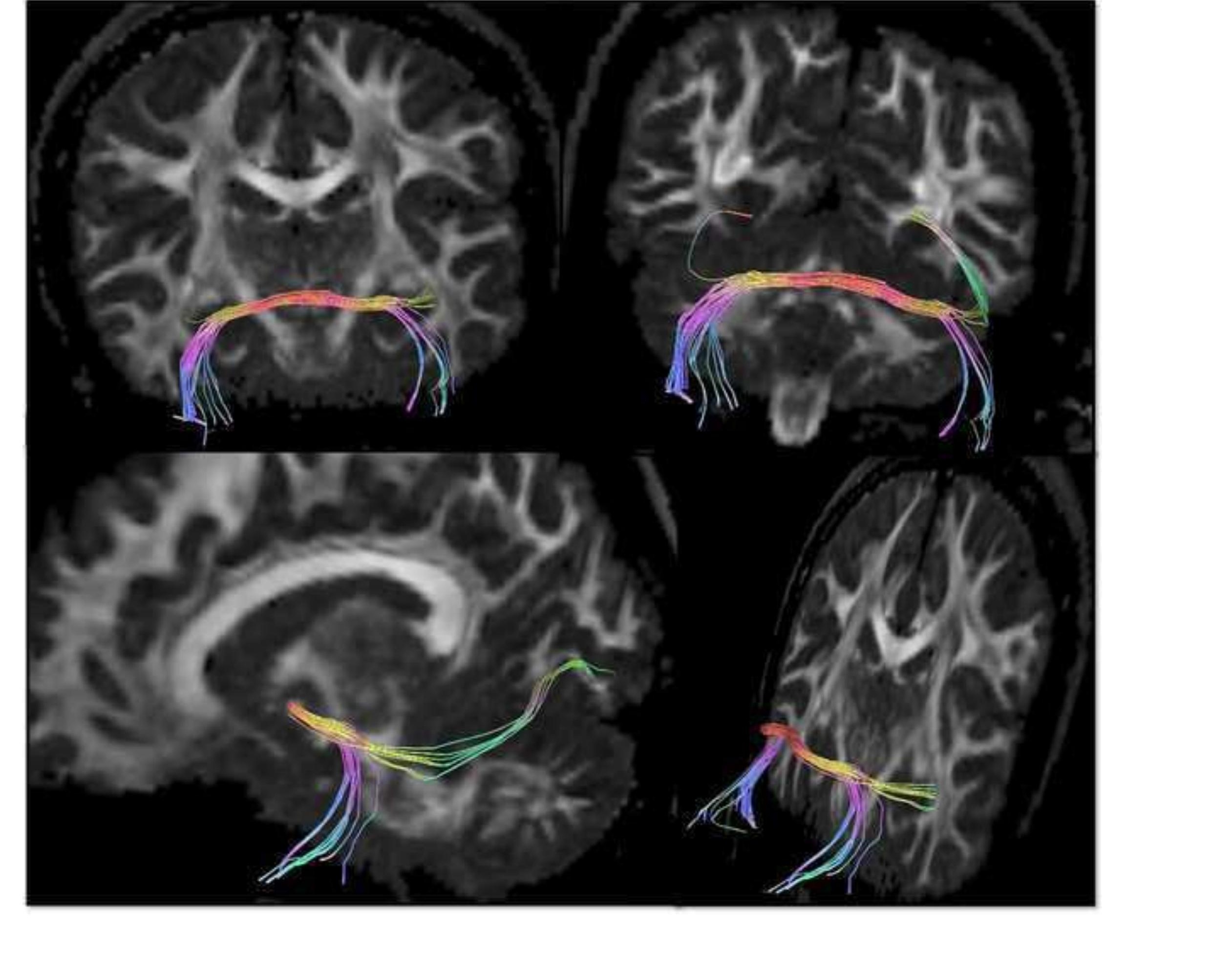

\section{(1)}

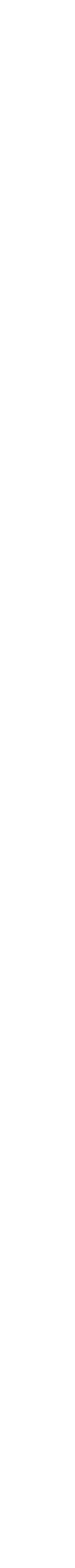

